\title{
Pseudo-Dirac neutrinos from flavour dependent CP symmetry
}

\author{
Anjan S. Joshipura ${ }^{a}$ and Ketan M. Patel ${ }^{b}$ \\ ${ }^{a}$ Physical Research Laboratory, \\ Navarangpura, Ahmedabad 380 009, India \\ ${ }^{b}$ Indian Institute of Science Education and Research Mohali, \\ Knowledge City, Sector 81, S.A.S. Nagar, Manauli 140 306, India \\ E-mail: anjan@prl.res.in, ketan@iisermohali.ac.in
}

ABSTRACT: Discrete residual symmetries and flavour dependent CP symmetries consistent with them have been used to constrain neutrino mixing angles and $\mathrm{CP}$ violating phases. We discuss here role of such CP symmetries in obtaining a pseudo-Dirac neutrino which can provide a pair of neutrinos responsible for the solar splitting. It is shown that if (a) $3 \times 3$ Majorana neutrino matrix $M_{\nu}$ is invariant under a discrete $Z_{2} \times Z_{2}$ symmetry generated by $S_{1,2}$, (b) CP symmetry $X$ transform $M_{\nu}$ as $X^{T} M_{\nu} X=M_{\nu}^{*}$, and (c) $X$ and $S_{1,2}$ obey consistency conditions $X S_{1,2}^{*} X^{\dagger}=S_{2,1}$, then two of the neutrino masses are degenerate independent of specific forms of $X, S_{1}$ and $S_{2}$. Explicit examples of this result are discussed in the context of $\Delta\left(6 n^{2}\right)$ groups which can also be used to constrain neutrino mixing matrix $U$. Degeneracy in two of the masses does not allow complete determination of $U$ but it can also be fixed once the perturbations are introduced. We consider explicit perturbations which break $Z_{2} \times Z_{2}$ symmetries but respect $\mathrm{CP}$. These are shown to remove the degeneracy and provide a predictive description of neutrino spectrum. In particular, a correlation $\sin 2 \theta_{23} \sin \delta_{C P}= \pm \operatorname{Im}[p]$ is obtained between the atmospheric mixing angle $\theta_{23}$ and the $\mathrm{CP}$ violating phase $\delta_{C P}$ in terms of a group theoretically determined phase factor $p$. Experimentally interesting case $\theta_{23}=\frac{\pi}{4}, \delta_{C P}= \pm \frac{\pi}{2}$ emerges for groups which predict purely imaginary $p$. We present detailed predictions of the allowed ranges of neutrino mixing angles, phases and the lightest neutrino mass for three of the lowest $\Delta\left(6 n^{2}\right)$ groups with $n=2,4,6$.

KEYwords: CP violation, Discrete Symmetries, Neutrino Physics

ARXIV EPRINT: 1805.02002 


\section{Contents}

1 Introduction 1

2 Degenerate neutrinos from GenCP 2

3 Degenerate pair of neutrinos in $\Delta\left(6 n^{2}\right)$ groups 5

$\begin{array}{lll}4 & \text { GenCP invariant perturbations } & 7\end{array}$

5 Numerical study of GenCP invariant perturbations 10

6 Summary 13

\section{Introduction}

Discrete flavour symmetries have been widely used in predicting the observed patterns of mixing among neutrinos, see [1-5] for reviews. Many of the successful schemes particularly those involving small discrete groups, predict a leading order mixing patterns which when perturbed can lead to the desired mixing in terms of very small unknown parameters. Celebrated examples are tri-bimaximal mixing, bi-maximal mixing or trimaximal mixing among neutrinos which provide a good description of neutrino mixing at the leading order. For the neutrino masses, a good leading order description is provided by a pair of degenerate neutrinos forming the pair responsible for the depletion of the solar neutrino flux. This leading order mass pattern can also be derived from discrete flavour symmetries $[6,7]$. See [8-10] for earlier works on two degenerate neutrinos.

Basic assumptions going in predictions of neutrino mixing are: (i) the leading order neutrino mass matrix is invariant under a residual $Z_{2} \times Z_{2}$ symmetry (ii) the charged lepton mass matrix $M_{l} M_{l}^{\dagger}$ is invariant under $Z_{m}$ with $m>2$ and (iii) both $Z_{2} \times Z_{2}$ and $Z_{m}$ are embedded in a discrete group $G_{f}$. The last condition determines the form of the generators of residual symmetries when leptons are assigned to a definite 3-dimensional irreducible representation of $G_{f}$. This in turn determines the exact form of the leptonic mixing matrix $U$ [11-15]. One can obtain a pair of degenerate neutrinos in this set up if the residual symmetry of the neutrino mass matrix is replaced by $Z_{n},(n>2)$ group with its generating element $S$ having the eigenvalues $\left(\eta, \eta^{*}, 1\right)$ with $\eta^{n}=1$ [7]. This automatically ensures degeneracy in two of the neutrino masses. Possible discrete subgroups of SU(3) having such $Z_{n}$ and the resulting mixing patterns among neutrinos have been extensively studied in [7]. We discuss here an alternative approach in which the occurrence of a pair of degenerate neutrinos is intimately linked to the imposition of flavour dependent CP symmetry. Flavour dependent CP symmetries have been widely studied in recent times [16-25] (see [26] for 
a recent review) with a view to constraint neutrino mixing matrix including Majorana neutrino phases which otherwise remain arbitrary.

In this article, we show that almost the same set up used for predicting mixing can also be used to obtain either partially degenerate or fully degenerate neutrinos spectrum with a judicious choice of the flavoured CP symmetry called Generalized CP (GenCP). The analysis presented here also uses the assumptions (i-iii) outlined above and thus is used also to constrain leptonic mixing parameters. Exact degeneracy in two of the masses makes one mixing angle, one Majorana phase and Dirac phase unphysical. The perturbations introduced to break degeneracy make these parameters physical and lead to predictions for these quantities which in general depend on the magnitude and nature of perturbations. We introduce a class of perturbations which are assumed to be invariant under GenCP. This results into a very predictive framework in which the predicted values of $\mathrm{CP}$ phases are almost independent of perturbations and mostly depend on the underlying symmetries only. We present some examples of these based on the $\Delta\left(6 n^{2}\right)$ groups. Neutrino mixing patterns resulting from the assumption of GenCP invariance have been derived earlier in case of the non-degenerate neutrinos in [19, 25, 27, 28].

We describe in the next section the general conditions under which GenCP symmetry leads to degeneracy in neutrino masses. The specific examples of such symmetries based on $\Delta\left(6 n^{2}\right)$ groups are discussed in section 3. In section 4 , we discuss a class of perturbations which lead to realistic masses and mixing pattern in a very predictive way and provide a numerical study of such perturbations in section 5. Finally, we summarize in section 6 .

\section{Degenerate neutrinos from GenCP}

We start with the conventional assumptions made in the symmetry based approaches.

(a) Assume that $3 \times 3$ Majorana neutrino mass matrix is invariant under a $Z_{2} \times Z_{2}$ symmetry generated by $S_{1}, S_{2}$ :

$$
S_{1,2}^{T} M_{\nu} S_{1,2}=M_{\nu}
$$

The above conditions togather imply $S_{3}^{T} M_{\nu} S_{3}=M_{\nu}$, where $S_{3}=S_{1} S_{2}$ is also an element of the $Z_{2} \times Z_{2}$ group. We also demand that the charged lepton mass matrix $M_{l}$ is invariant under a $Z_{m}$ transformation generated by $T_{l}$ such that

$$
T_{l}^{\dagger} M_{l} M_{l}^{\dagger} T_{l}=M_{l} M_{l}^{\dagger} .
$$

(b) $M_{\nu}$ is invariant under GenCP $X$ :

$$
X^{T} M_{\nu} X=M_{\nu}^{*} .
$$

(c) CP transformation $X$ followed by a $Z_{2} \times Z_{2}$ transformation and an inverse CP transformation is equivalent to a $Z_{2} \times Z_{2}$ transformation on fields and operators. This requires that $X$ maps $Z_{2} \times Z_{2}$ groups to itself [16, 17, 20]. This can happen in three distinct ways. Either

$$
X S_{i}^{*} X^{\dagger}=S_{i}
$$


or

$$
X S_{i}^{*} X^{\dagger}=S_{j}, \quad X S_{j}^{*} X^{\dagger}=S_{i} \text { and } X S_{k}^{*} X^{\dagger}=S_{k},
$$

or

$$
X S_{i}^{*} X^{\dagger}=S_{j}, \quad X S_{j}^{*} X^{\dagger}=S_{k} \quad \text { and } X S_{k}^{*} X^{\dagger}=S_{i},
$$

where $i, j, k=1,2,3$ and $i \neq j \neq k$. No specific forms for $X$ and $S_{i}$ are assumed here except that they are unitary and $S_{i}^{2}=\mathbf{1}, S_{i} S_{j}=S_{j} S_{i}$, Det. $S_{i}=1$. The conditions in eqs. (2.4), (2.5), (2.6) are special cases of general consistency conditions which are needed to be satisfied for consistent definition of GenCP in a discrete group $G_{f}[16,17,29-32]$ :

$$
X_{r} \rho_{r}^{*}(g) X_{r}^{\dagger}=\rho_{r}\left(g^{\prime}\right),
$$

where $X_{r}$ is GenCP in representation $\rho_{r}$ of $G_{f}$ and $g, g^{\prime} \in G_{f}$.

We now show that neutrino mass degeneracy directly follows from these basic assumptions. Specifically, we prove the following result:

If eqs. (2.1), (2.3) are satisfied and $X$ maps $Z_{2} \times Z_{2}$ to itself according to eq. (2.5) (eq. (2.6)) then the absolute masses of two (all three) neutrinos are equal.

The proof goes as follows. For simplicity, we consider a specific case with $i=1, j=2$ and $k=3$ in eq. (2.5). Let $U_{S}$ be a common matrix diagonalizing the commuting matrices $S_{1,2}$ :

$$
U_{S}^{\dagger} S_{1} U_{S}=\text { Diag. }(1,-1,-1) \equiv d_{1}, \quad U_{S}^{\dagger} S_{2} U_{S}=\text { Diag. }(-1,1,-1) \equiv d_{2} .
$$

The $d_{1,2}$ represent sets of eigenvalues of $Z_{2}$ generators $S_{1,2}$. We have chosen a specific ordering in writing above equations. The other ordering would give results which can be obtained by permutations of the one derived here. Eqs. (2.1), (2.8) imply

$$
d_{1,2} D=D d_{1,2}
$$

with

$$
D=U_{S}^{T} M_{\nu} U_{S}
$$

This implies $D=$ Diag. $\left(m_{1}, m_{2}, m_{3}\right)$. The masses $m_{i}$ are in general complex. The CP transformation matrix in the diagonal basis of $M_{\nu}$ becomes

$$
\tilde{X}=U_{S}^{\dagger} X U_{S}^{*}
$$

and the constraint in eq. (2.5) with $i=1, j=2$ and $k=3$ then implies

$$
\tilde{X} d_{1,2}=d_{2,1} \tilde{X}
$$

Using the explicit form of $d_{1,2}$ as given in eq. (2.8), one finds that the most general $\tilde{X}$ satisfying eqs. (2.12) is given by

$$
\tilde{X}=\left(\begin{array}{ccc}
0 & p_{1} & 0 \\
p_{2} & 0 & 0 \\
0 & 0 & p_{3}
\end{array}\right)
$$


where $p_{i}$ are arbitrary phases. Eq. (2.3) can be rewritten using eqs. (2.10), (2.11) as

$$
\tilde{X}^{T} D \tilde{X}=D^{*}
$$

Eqs. (2.13), (2.14) give:

$$
\frac{m_{2}^{*}}{m_{1}}=p_{1}^{2}=p_{2}^{2}, \frac{m_{3}^{*}}{m_{3}}=p_{3}^{2} .
$$

Since $p_{1,2}$ are phases, eq. (2.15) immediately leads to degeneracy of two masses

$$
\left|m_{1}\right|=\left|m_{2}\right|
$$

proving the above assertion.

Following the similar reasoning, it is easy to verify that a choice $i=2, j=3, k=1$ in eq. (2.5) leads to $\left|m_{2}\right|=\left|m_{3}\right|$ while $i=3, j=1, k=2$ in the same equation would imply $\left|m_{1}\right|=\left|m_{3}\right|$. On the other hand, if eq. (2.6) is true then the same reasoning as above leads to

$$
\tilde{X}=\left(\begin{array}{ccc}
0 & 0 & p_{1} \\
p_{2} & 0 & 0 \\
0 & p_{3} & 0
\end{array}\right) \quad \text { or } \quad \tilde{X}=\left(\begin{array}{ccc}
0 & p_{1} & 0 \\
0 & 0 & p_{2} \\
p_{3} & 0 & 0
\end{array}\right)
$$

Substitution of either of the above $\tilde{X}$ in eq. (2.14) leads to equality of the absolute masses of all three neutrinos. In the following, we confine ourselves to discussion of partially degenerate spectrum.

Apart from implying degeneracy, eq. (2.14) also fixes phases of two of the masses in terms of $p_{1,2,3}$. The latter are determined by the structure of $X$ and would follow once the forms of $S_{1,2}$ and the underlying discrete groups are fixed. Explicitly, let $m_{1}=m e^{i \alpha}$ with $m$ real and positive. Then

$$
m_{2}=m e^{-i \alpha} p_{1}^{-2}, m_{3}=m^{\prime} p_{3}^{-1} .
$$

Defining

$$
P_{\nu}=\operatorname{Diag} .\left(e^{-\frac{i \alpha}{2}}, e^{\frac{i \alpha}{2}} p_{1}, p_{3}^{\frac{1}{2}}\right)
$$

$U_{R}=U_{S} P_{\nu}$ makes the neutrino mass matrix diagonal with real positive ${ }^{1}$ entries. Now using eq. (2.10) one gets

$$
U_{R}^{T} M_{\nu} U_{R} \equiv \text { Diag. }\left(m, m, m^{\prime}\right) .
$$

In this basis, $\tilde{X}_{R} \equiv U_{R}^{\dagger} X U_{R}^{*}$ has the form

$$
\tilde{X}_{R}=\left(\begin{array}{ccc}
0 & 1 & 0 \\
(-1)^{l} & 0 & 0 \\
0 & 0 & 1
\end{array}\right)
$$

with $l=0,1$ as can be explicitly checked using eqs. (2.13), (2.18). It is remarkable that there are only two possible forms of $\tilde{X}_{R}$ irrespective of the underlying discrete group or

\footnotetext{
${ }^{1}$ We are assuming $m^{\prime}$ to be positive. If not, it can be made positive by redefining the $(3,3)$ element of $P_{\nu}$.
} 
the chosen residual symmetry. This result follows from the required consistency equations in a model independent manner. The other possible $\tilde{X}_{R}$ are either related to the above by permutations or by an overall change of sign. $\tilde{X}_{R}$ with $l=0$ is symmetric and hence the corresponding $X$ is symmetric. On the other hand $X$ in case of $l=1$ is neither symmetric or antisymmetric.

Conversely, if we assume two of the neutrinos to be degenerate then one could argue that the allowed forms of $\tilde{X}_{R}$ are still given by eq. (2.20). Going to the basis with real and positive masses, eq. (2.14) becomes

$$
\tilde{X}_{R} D_{R}=D_{R} \tilde{X}_{R}
$$

where $D_{R}$ is a diagonal matrix with real and positive entries given by $\left|m_{i}\right|$. If we assume $\left|m_{1}\right|=\left|m_{2}\right|$ the allowed forms of $\tilde{X}_{R}$ satisfying above equations are given by

$$
\tilde{X}_{R}=\left(\begin{array}{ccc}
\cos \theta & \sin \theta & 0 \\
-\sin \theta & \cos \theta & 0 \\
0 & 0 & \pm 1
\end{array}\right) ; \quad \tilde{X}_{R}=\left(\begin{array}{ccc}
\cos \theta & \sin \theta & 0 \\
\sin \theta & -\cos \theta & 0 \\
0 & 0 & \pm 1
\end{array}\right) \text {. }
$$

The above form of $\tilde{X}_{R}$ implies existence of an unbroken $O(2)$ symmetry in the neutrino mass basis. The consistency conditions $\tilde{X}_{R} d_{1,2}=d_{2,1} \tilde{X}_{R}$ which follow from eq. (2.12) and definition of $\tilde{X}_{R}$ lead to $\theta=\frac{\pi}{2}$ in both the cases and the allowed forms of $\tilde{X}_{R}$ reduce to eq. (2.20). The consistency conditions break the full $O(2)$ symmetry into its discrete subgroups, $Z_{2}$ and $Z_{4}$, generated by $\tilde{X}_{R}$ in eq. (2.20) for $l=0$ and $l=1$ respectively. We note that:

- Degeneracy here follows from eq. (2.5). If $Z_{2} \times Z_{2}$ is mapped by $X$ instead according to eq. (2.4) then it is easy to show that the corresponding $\tilde{X}_{R}$ is proportional to an identity matrix instead of non-diagonal $\tilde{X}_{R}$ as in eq. (2.20). In that case, eq. (2.3) does not imply any restriction on the neutrino masses.

- A class of discrete symmetric groups leading to a degenerate mass spectrum were identified and studied in [7] by requiring that these groups posses a generator $S$ with eigenvalues $\left(\eta, \eta^{*}, 1\right)$, where $\eta^{m}=1$ and $m>2$. The chosen form of $S$ corresponds to non-trivial discrete subgroups of $O(2)$ which ensures degeneracy in the masses of the first two generations of neutrinos. The mechanism for obtaining degenerate spectrum through CP symmetry presented here appears logically different but invariance under $S$ would nevertheless be present since any neutrino mass matrix with two degenerate eigenvalues is always invariant in a suitable basis under a symmetry generated by $S$. This symmetry thus is an effective symmetry in this approach.

In the next section, we discuss specific groups and CP symmetries as concrete realization of above general discussions.

\section{Degenerate pair of neutrinos in $\Delta\left(6 n^{2}\right)$ groups}

The $\Delta\left(6 n^{2}\right)$ as possible flavour symmetry groups are widely studied [33-35]. In particular, all possible $\mathrm{CP}$ and residual neutrino $Z_{2} \times Z_{2}$ symmetries in $\Delta\left(6 n^{2}\right)$ have been extensively 
studied with a view to constrain neutrino Majorana phases [20]. Here we show that a subclass of CP symmetries identified in [20] actually satisfy the conditions of theorem derived in the previous section and hence lead to a degenerate pair of neutrinos. For this purpose, we closely follow the notation of [20,34].

The groups in $\Delta\left(6 n^{2}\right)$ series can be constructed from three generators $a, b, c$ satisfying

$$
a^{3}=b^{2}=(a b)^{2}=c^{n}=1 .
$$

It is convenient to define $d=a^{2} c a$ satisfying $a c a^{2}=c^{-1} d^{-1}$. We shall be using a specific three dimensional representation for these:

$$
a=\left(\begin{array}{lll}
0 & 1 & 0 \\
0 & 0 & 1 \\
1 & 0 & 0
\end{array}\right), b=-\left(\begin{array}{lll}
0 & 0 & 1 \\
0 & 1 & 0 \\
1 & 0 & 0
\end{array}\right), c=\left(\begin{array}{ccc}
\eta & 0 & 0 \\
0 & \eta^{*} & 0 \\
0 & 0 & 1
\end{array}\right), d=\left(\begin{array}{ccc}
1 & 0 & 0 \\
0 & \eta & 0 \\
0 & 0 & \eta^{*}
\end{array}\right),
$$

with $\eta^{n}=1$. We consider specific Klein groups $K=\left(1, c^{\frac{n}{2}}, a b c^{\gamma}, a b c^{\gamma+\frac{n}{2}}\right)$ with $\gamma=$ $0,1, \ldots, \frac{n}{2}-1$ which are obtained as subgroups of an underlying $\Delta\left(6 n^{2}\right)$ group with only even values for $n$. Of these, we specifically choose the neutrino symmetries as

$$
S_{1}=a b c^{\gamma}=\left(\begin{array}{ccc}
0 & -\eta^{-\gamma} & 0 \\
-\eta^{\gamma} & 0 & 0 \\
0 & 0 & -1
\end{array}\right) ; \quad S_{2}=a b c^{\gamma+\frac{n}{2}}=\left(\begin{array}{ccc}
0 & \eta^{-\gamma} & 0 \\
\eta^{\gamma} & 0 & 0 \\
0 & 0 & -1
\end{array}\right)
$$

The allowed set of CP symmetries satisfying the consistency conditions are also identified in $[20]$ as

$$
\left(X_{1}, X_{2}, X_{3}, X_{4}\right) \equiv\left(c^{x} d^{2 \gamma+2 x}, a b c^{x} d^{2 x}, c^{x} d^{2 x+2 \gamma+\frac{n}{2}}, a b c^{x} d^{2 x+2 \gamma+\frac{n}{2}}\right),
$$

where $x=0,1, \ldots, n-1$ and we have neglected an overall phase. All the four $X_{i}$ map the Klein group into itself and $X_{3,4}$ satisfy eq. (2.5):

$$
X_{3,4} S_{1,2}^{*} X_{3,4}^{\dagger}=S_{2,1}
$$

This can be verified from the explicit 3-dimensional representation in eq. (3.2) and the form of $X_{3,4}$ :

$$
X_{3}=\left(\begin{array}{ccc}
\eta^{x} & 0 & 0 \\
0 & -\eta^{x+2 \gamma} & 0 \\
0 & 0 & -\eta^{-2 x-2 \gamma}
\end{array}\right) ; \quad X_{4}=\left(\begin{array}{ccc}
0 & \eta^{x} & 0 \\
-\eta^{x} & 0 & 0 \\
0 & 0 & \eta^{-2 x}
\end{array}\right)
$$

Both these symmetries must lead to a degenerate pair. This is explicit from the construction of neutrino mass matrices satisfying eqs. (2.1), (2.3) with $X$ as $X_{3}$ and $X_{4}$. In case of $X_{3}$ one gets

$$
M_{0 \nu}=m_{0}\left(\begin{array}{ccc}
A \eta^{-x} & i B \eta^{-x-\gamma} & 0 \\
i B \eta^{-x-\gamma} & A \eta^{-x-2 \gamma} & 0 \\
0 & 0 & C \eta^{2 x+2 \gamma}
\end{array}\right)
$$


Here $A, B, C$ are required to be real. For $X_{4}$, imposition of eq. (2.3) leads to the following form:

$$
M_{0 \nu}=m_{0}\left(\begin{array}{ccc}
A \eta^{-x} & i B \eta^{-x} & 0 \\
i B \eta^{-x} & A \eta^{-x} & 0 \\
0 & 0 & C \eta^{2 x}
\end{array}\right),
$$

with real $A, B, C$. These matrices lead to a degenerate pair. ${ }^{2}$ It is to be noted that just the generalized CP invariance alone is sufficient to give degeneracy in case of $X_{4}$. Imposition of the residual symmetries $S_{1,2}$ invariance further leads to $A=0$ in eq. (3.6) for $\gamma \neq 0$ in $S_{1,2}$. For $\gamma=0$ in $S_{1,2}$, eq. (3.6) is also invariant under $S_{1,2}$.

\section{GenCP invariant perturbations}

The residual Klein symmetry along with particular $Z_{m}$ symmetry of $M_{l} M_{l}^{\dagger}$ lead to prediction of vanishing Dirac CP phase $[34,35]$ in $\Delta\left(6 n^{2}\right)$ groups. The additional use of GenCP further lead to predictions of the Majorana phases [20]. These predictions hold strictly only for the non-degenerate neutrino masses and would not be true in cases of specific CP symmetries $X_{3,4}$ which imply degeneracy since in this case Dirac CP and one of the Majorana CP phases can be rotated away. One therefore needs perturbations which break degeneracy. We consider here a very specific class of perturbations which break the residual $Z_{2} \times Z_{2}$ symmetry of $M_{\nu}$ but preserve the GenCP invariance. As we will show, this class of perturbations lead to a departure from degeneracy but lead to very definite prediction of Dirac CP phase in terms of the atmospheric mixing angle irrespective of the values of perturbing parameters.

We assume the neutrino mass matrix $M_{\nu}$ to be

$$
M_{\nu}=M_{0 \nu}+\delta M_{\nu} .
$$

Here, $M_{0 \nu}$ is assumed to satisfy eqs. (2.1), (2.3) and thus has the form given in eq. (3.5). $\delta M_{\nu}$ only satisfies eq. (2.3):

$$
X_{3}^{T} \delta M_{\nu} X_{3}=\delta M_{\nu}^{*} .
$$

We do not consider here $X_{4}$ since in this case the most general matrix $M_{\nu}$ invariant under $X_{4}$ alone leads to degeneracy as shown in the earlier section. Types of perturbations considered here are thus not sufficient to give correct neutrino mass spectrum in case of $X_{4}$.

The explicit form of $X_{3}$ given in eq. (3.4) leads to the following general neutrino mass matrix:

$$
M_{\nu}=m_{0}\left(\begin{array}{ccc}
A \eta^{-x} & i B \eta^{-x-\gamma} & i \epsilon_{1} \eta^{\gamma+\frac{x}{2}} \\
i B \eta^{-x-\gamma} & A\left(1+\epsilon_{3}\right) \eta^{-x-2 \gamma} & \epsilon_{2} \eta^{\frac{x}{2}} \\
i \epsilon_{1} \eta^{\gamma+\frac{x}{2}} & \epsilon_{2} \eta^{\frac{x}{2}} & C \eta^{2 x+2 \gamma}
\end{array}\right)
$$

Here $\epsilon_{1,2,3}$ are required to be real. We have also redefined $A, B, C$ to absorb some of the redundant parameters implied by perturbations. As seen from above, the general

\footnotetext{
${ }^{2}$ Neutrino mass matrices invariant under $X_{3,4}$ and $S_{1,2}$ were earlier derived in [20]. These matrices did not contain a relative factor of $i$ present here between the off-diagonal and diagonal elements in eqs. (3.5), (3.6). This factor leads to degeneracy which should occur in these cases as argued on general grounds.
} 
perturbations in this case are characterized by three real parameters. It was shown in $[25$, 28 ] that GenCP invariant perturbations in case of $X_{1,2}$ can be described by an orthogonal matrix containing three angles. This is true in this case also. We notice that $M_{\nu}$ in eq. (4.3) can be made real by multiplying it with a diagonal phase matrix $P$ :

$$
\begin{gathered}
P=\text { Diag. }\left(\eta^{\frac{x}{2}},-i \eta^{\frac{x}{2}+\gamma}, i \eta^{-x-\gamma}\right), \\
P^{T} M_{\nu} P=m_{0}\left(\begin{array}{ccc}
A & B & -\epsilon_{1} \\
B & -A\left(1+\epsilon_{3}\right) & \epsilon_{2} \\
-\epsilon_{1} & \epsilon_{2} & -C
\end{array}\right) .
\end{gathered}
$$

This matrix being real and symmetric, can be diagonalized by an orthogonal matrix. It follows from eqs. (4.3), (4.5) that the matrix diagonalizing $M_{\nu}$ has the form

$$
U_{\nu}=P O K
$$

where $O$ is a real orthogonal matrix which diagonalizes the matrix, Eq. (4.5) and $K$ is a diagonal matrix with elements \pm 1 or $\pm i$ which is introduced to make the eigenvalues of $M_{\nu}$ positive. The leptonic mixing matrix is given by

$$
U_{\mathrm{PMNS}} \equiv U=U_{l}^{\dagger} U_{\nu}=U_{l}^{\dagger} P O K \text {. }
$$

Here $U_{l}$ can be determined from eq. (2.2) in terms of the matrix which diagonalize $T_{l}$. A diagonal $T_{l}$ implies a trivial $U_{l}$ which leads to vanishing $\theta_{23}$ and $\theta_{13}$ in the absence of perturbations as can be seen from eqs. (3.5), (4.6), (4.7). Viable $\theta_{23}$ would then require large corrections and therefore we do not consider this case. There exist two classes of non-diagonal $Z_{m}$ in $\Delta\left(6 n^{2}\right)$ which can be chosen as $T_{l}$. These have the general form in the basis defined by eq. (3.1):

$$
T_{l}=\left(\begin{array}{ccc}
0 & \eta_{1} & 0 \\
0 & 0 & \eta_{2} \\
\eta_{3} & 0 & 0
\end{array}\right) \quad \text { or } T_{l}=-\left(\begin{array}{ccc}
0 & 0 & \eta_{1} \\
0 & \eta_{3} & 0 \\
\eta_{2} & 0 & 0
\end{array}\right),
$$

with $\eta_{1} \eta_{2} \eta_{3}=1$. The first set of $T_{l}$ is chosen as a proper symmetry in the discussion of non-degenerate neutrinos $[20,34,35]$. Here we argue that the second type of $T_{l}$ or its permutations are the only viable choices if we assume that perturbations are small and their role is to provide small corrections to zeroeth order mixing. For this, we note that $O$ is a block diagonal matrix with $O_{a 3}, O_{3 a}$ zero for $a=1,2$ in the absence of perturbations, see the form of $M_{0 \nu}$, eq. (3.5). The third column of $O$ is thus given by $\psi=(0,0,1)^{T}$ and this represents the eigenvector for the non-degenerate mass. It then follows that $U_{l}^{\dagger} \psi$ should provide a good leading order approximation to the third column of $U$. If $T_{l}$ is chosen as the first set of matrix then the absolute value of all the elements of $U_{l}$ is $\frac{1}{\sqrt{3}}$ and the $\left|U_{l}^{\dagger} \psi\right|$ is also democratic implying $\sin ^{2} \theta_{13}=\frac{1}{3}$. This would require large corrections. If $T_{l}$ is chosen in the second category, then the corresponding $U_{l}$ can be written as

$$
U_{l}=\frac{1}{\sqrt{2}}\left(\begin{array}{ccc}
0 & 1 & -\eta_{1} \lambda^{*} \\
\sqrt{2} & 0 & 0 \\
0 & \lambda \eta_{1}^{*} & 1
\end{array}\right)
$$


Here, $\lambda=\left(\eta_{1} \eta_{2}\right)^{\frac{1}{2}}$ and three columns of above $U_{l}$ correspond to eigenvectors with eigenvalues $-\lambda^{* 2}, \lambda,-\lambda$ respectively. Given this $U_{l},\left|U_{l}^{\dagger} \psi\right|$ is given by $\frac{1}{\sqrt{2}}(0,1,1)$ corresponding to vanishing (maximal) $\theta_{13}\left(\theta_{23}\right)$ at the leading order. This can get corrected to the desired values after small perturbations.

If neutrinos are non-degenerate, then above $U_{l}$ alongwith $S_{1,2}$ given in eq. (3.2) leads to bi-maximal mixing pattern as long as $T_{l}$ is not $Z_{2}$. This predicts maximal solar angle which would need to be corrected by large perturbations. In the present case, solar angle remains undetermined at the leading order due to degeneracy and could get corrected by small perturbations.

One can construct the mixing matrix $U$ using $U_{\nu}$ and $U_{l}$ respectively from eqs. (4.6) and (4.9). The elements of $U$ are given as follows:

$$
\begin{aligned}
U_{e j} & =-i \eta^{\gamma+\frac{x}{2}} O_{2 j}, \\
U_{\mu j} & =\frac{\eta^{\frac{x}{2}}}{\sqrt{2}}\left(O_{1 j}+p O_{3 j}\right), \\
U_{\tau j} & =\frac{-\lambda \eta_{1}^{*} \eta^{\frac{x}{2}}}{\sqrt{2}}\left(O_{1 j}-p O_{3 j}\right),
\end{aligned}
$$

where $j=1,2,3$ and $p=i \lambda^{*} \eta_{1} \eta^{-\gamma-\frac{3 x}{2}}$. By comparing above $U$ with standard parameterization of it as given by [36] one arrives at the following relations:

$$
\begin{aligned}
\sin 2 \theta_{23} \sin \delta_{C P} & = \pm \operatorname{Im}[p] \\
\sin \alpha_{21} & =\sin \left(\alpha_{31}-2 \delta_{C P}\right)=0 \\
\cos 2 \theta_{23} & =\sin \chi\left(1-\operatorname{Im}[p]^{2}\right)^{\frac{1}{2}}
\end{aligned}
$$

where $\tan \chi=\frac{O_{13}}{O_{33}}$. It is seen from the first two equations that the $\mathrm{CP}$ violating phases are predicted solely in terms of a group theoretical phase $p$ and $\theta_{23}$. The latter depends upon perturbation if $p$ is not purely imaginary. One obtains the $\mu-\tau$ reflection symmetry [37-44] (see also [45] for a recent review) results

$$
\pm \delta_{C P}=2 \theta_{23}=\frac{\pi}{2}, \quad \sin \alpha_{21}=\sin \alpha_{31}=0
$$

for special class of residual symmetries for which $p$ is purely imaginary. While this symmetry is close to the observed values there already exists hints on its small breaking since the best fit values of $\theta_{23}$ is found to be different from the maximal values for both the orderings in neutrino masses [46]. For $n=2$, the group $\Delta\left(6 \times 2^{2}\right) \equiv S_{4}$ leads to either real or pure imaginary $p$ as can be seen from figure 1 . The first of these implies $\delta_{\mathrm{CP}}=0$ while the latter leads to residual unbroken $\mu-\tau$ reflection symmetry even in the presence of perturbations as discussed above. Groups with higher $n$ contain other values of $p$ in additions to the ones in $S_{4}$. For example, $n=4$ has $\operatorname{Im}[p]= \pm \frac{1}{\sqrt{2}}$ and $n=6$ contains $\operatorname{Im}[p]= \pm \frac{1}{2}$ and $\pm \frac{\sqrt{3}}{2}$. These values may be taken as approximate predictions of $\sin \delta_{C P}$ since the allowed $3 \sigma$ range for $\sin 2 \theta_{23}$ is quite narrow (0.97-1.0) [46] and hence $\sin \delta_{C P}$ is nearly close to $\operatorname{Im}[p]$. The exact values depend on details of perturbations. We study these in the next section. 


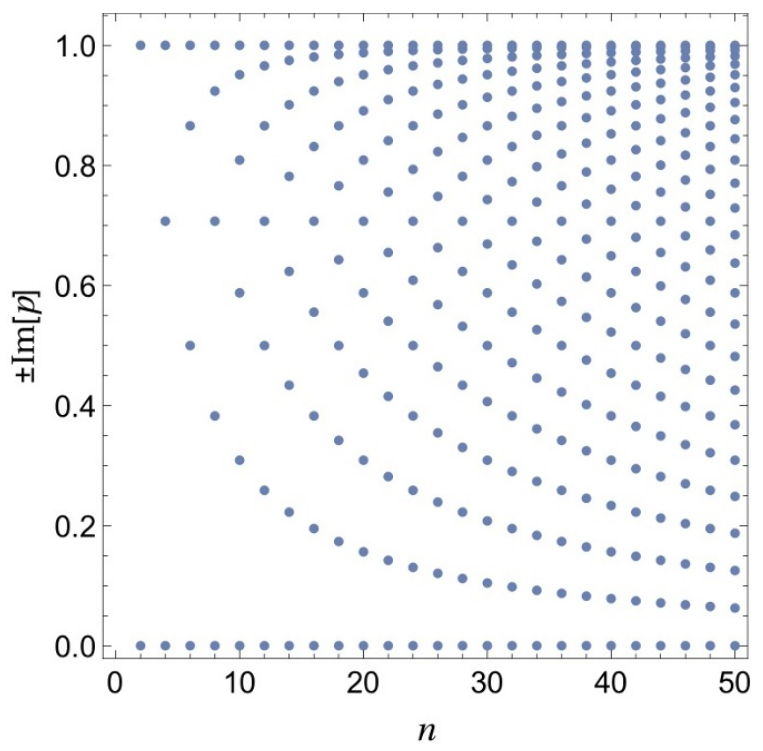

Figure 1. The values of $\pm \operatorname{Im}[p]$ predicted by the groups of $\Delta\left(6 n^{2}\right)$ series for even $n$, and $n \leq 50$.

We note that correlation similar to the first of eq. (4.11) was also derived in [37] by imposing a specific CP symmetry on the neutrino mass matrix in the flavour basis. Imposing our CP symmetry $X_{3}$ in symmetry basis is equivalent to imposing a symmetry $X_{3 f} \equiv U_{l}^{\dagger} X_{3} U_{l}^{*}$ in the flavour basis with diagonal charged lepton mass matrix, where $U_{l}$ is given by eq. (4.9). Explicitly

$$
X_{3 f}=\left(\begin{array}{ccc}
\eta^{x+2 \gamma} & 0 & 0 \\
0 & e^{i \beta} \cos \theta & i e^{i \frac{\beta+\alpha}{2}} \sin \theta \\
0 & i e^{i \frac{\beta+\alpha}{2}} \sin \theta & e^{i \alpha} \cos \theta
\end{array}\right)
$$

where $\sin \theta=\operatorname{Im}[p]=\operatorname{Im}\left[i \lambda^{*} \eta_{1} \eta^{-\gamma-\frac{3 x}{2}}\right], e^{i \beta}=\lambda^{*} \eta_{1} \eta^{-x / 2-\gamma}$ and $e^{i \alpha}=\lambda \eta_{1}^{*} \eta^{-x / 2-\gamma}$. This has the same form as the generalized $\mu-\tau$ reflection symmetry introduced in [37] and correlation derived by them coincide with our eq. (4.11) with an important difference. $\theta, \beta$, $\gamma$ introduced by them are arbitrary parameters while here they are determined by group theory. As a result, one predicts definite pattern of the $\mu$ - $\tau$ symmetry breaking since the phase factor $p$ takes specific discrete values based on the chosen group and residual symmetries. The values of real and imaginary parts of $p$ predicted by the first few groups of the group series $\Delta\left(6 n^{2}\right)$ which possess 3 -dimensional irreducible representations are displayed in figure 1 . We have displayed only the groups with even $n$ since the groups with odd $n$ do not contain any Klein groups as subgroups.

\section{$5 \quad$ Numerical study of GenCP invariant perturbations}

The perturbations to $M_{0 \nu}$ characterized by eq. (4.2) are also expected to induce viable solar neutrino mass scale. We investigate this by performing numerical study of the neutrino 

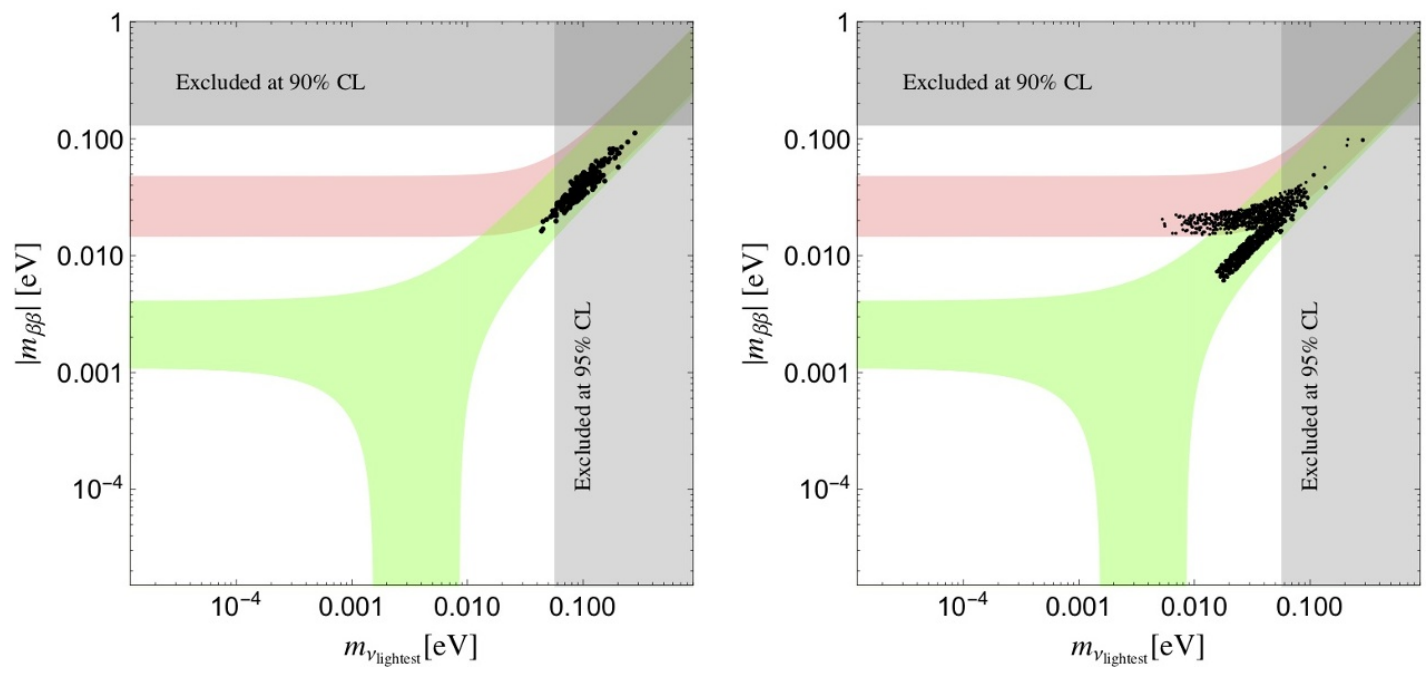

Figure 2. Correlations between the effective neutrinoless double beta decay mass and the lightest neutrino mass in case of $n=2$ (with $\gamma=0, x=-\eta_{1}=\eta_{2}=1$ ). The left (right) panel corresponds to $\epsilon_{\max }=0.02(0.1)$. The black points in both the panels are in agreement with the results of a global fit [46] of neutrino oscillation parameters within $3 \sigma$.

mass matrix $M_{\nu}$ given in eq. (4.3). The parameters $A, B$ and $C$ are chosen from uniform random distributions of real numbers in the range from \pm 0.1 to \pm 1.0 . The perturbation parameters are also randomly chosen such that $\left|\epsilon_{i}\right| \leq \epsilon_{\max }$ where $i=1,2,3$ and we study cases for $\epsilon_{\max }=0.02$ or 0.1 . With this choice, it is ensured that the magnitude of perturbation parameters is always smaller than that of $A, B$ and $C$. Several sample points are generated and for each point the parameter $m_{0}$ in eq. (4.3) is determined using the known value of atmospheric squared mass difference, $\Delta m_{31}^{2}=2.494 \times 10^{-3} \mathrm{eV}^{2}\left(\Delta m_{23}^{2}=2.465 \times 10^{-3} \mathrm{eV}^{2}\right)$ in case of normal (inverted) ordering in the neutrino masses. Here, $\Delta m_{i j}^{2} \equiv m_{i}^{2}-m_{j}^{2}$ and we use the observed values from the latest global fit results NuFIT 3.2 (2018) [46]. We then compute neutrino masses and mixing using numerical $M_{\nu}$ and $U_{l}$ given in eq. (4.9). The results obtained for some small groups in $\Delta\left(6 n^{2}\right)$ group series corresponding to $n=2,4,6$ are displayed in figures $2-4$.

The correlations between the effective mass of neutrinoless double beta decay $\left|m_{\beta \beta}\right|$ and the lightest neutrino mass are displayed in figure 2 in case of $S_{4}$ group and for small and large perturbations. The region shown by the horizontal gray band is disfavoured by the combined limit obtained from different neutrinoless double beta decay experiments at $90 \%$ confidence level [47]. The vertical gray band represents the region excluded by the limit on the sum of neutrino masses obtained from the latest results from PLANCK experiment, baryon acoustic oscillations and type Ia supernovae [48]. The regions shaded by green and red colours are generically allowed when the Dirac and Majorana phases are unconstrained in case of normal and inverted ordering in neutrino masses respectively. The scattered points in figure 2 are in agreement with the $3 \sigma$ ranges of global fit [46] values of solar and atmospheric mass differences and all three mixing angles. It can be seen that the small values of perturbations, corresponding to $\epsilon_{\max }=0.02$, require nearly 

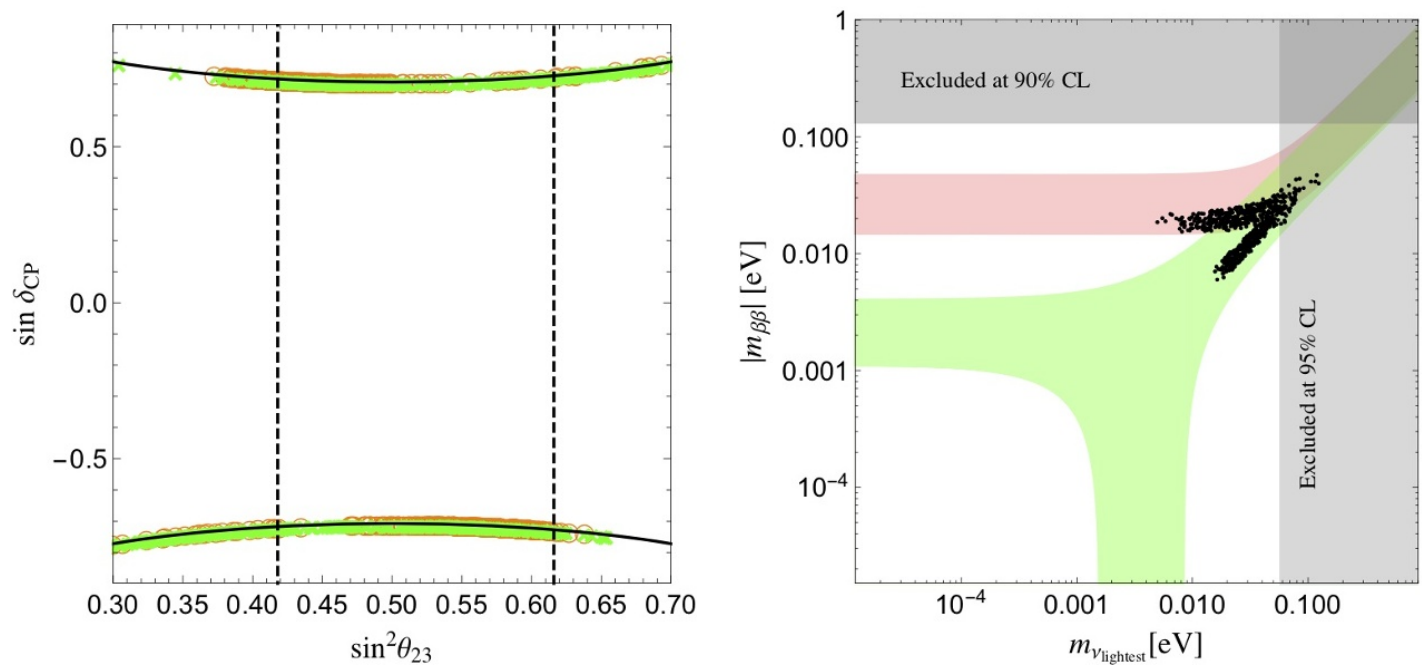

Figure 3. The results for $n=4$ (with $\gamma=0, x=-\eta_{1}=\eta_{2}=1$ ) and $\epsilon_{\max }=0.1$. In the left panel, the orange circles (green crosses) correspond to the normal (inverted) ordering in the neutrino masses. All these points are in agreement with global fit values of $\Delta m_{21}^{2}, \Delta m_{31}^{2}, \theta_{12}$ and $\theta_{13}$ within $3 \sigma$. The region between the vertical dashed lines correspond to experimentally allowed $3 \sigma$ range of $\sin ^{2} \theta_{23}$. The black contours represent the correlation given by the first of eq. (4.11). In the right panel, all the points are in agreement with the results of global fit of neutrino oscillation data within $3 \sigma$.

degenerate mass spectrum for all the three neutrinos which is almost disfavoured by the considered limit on the sum of neutrino masses. Increasing the magnitude of perturbations helps in evading this constraint. However one obtains lower limit on the lightest neutrino mass: $m_{\nu_{1}}>0.015 \mathrm{eV}$ for normal ordering and $m_{\nu_{3}}>0.005 \mathrm{eV}$ for inverted ordering if the magnitude of perturbation is smaller than that of the leading order parameters. This case predicts maximal values for $\theta_{23}$ and $\delta_{\mathrm{CP}}$ and vanishing Majorana phases irrespective of the magnitude of perturbation and therefore it is easily falsifiable in many ways.

We also perform similar numerical investigations for some cases which provide alternative predictions for $\theta_{23}$ and $\delta_{\mathrm{CP}}$. We choose the groups in $\Delta\left(6 n^{2}\right)$ series corresponding to $n=4,6$. As it can be seen from figure 1 , this choice offer three distinct values for $p$, one for $n=4$ and two for $n=6$, which are neither real nor imaginary. In these cases the predicted value of $\theta_{23}$ depends on the perturbations and the values of CP phases are determined by the correlation predicted in the first two of eq. (4.11). The results are displayed in figure 3 for $n=4$ and in figure 4 for $n=6$. It is noticed that correlation between $\left|m_{\beta \beta}\right|$ and the lightest neutrino mass remains almost similar in all these cases.

There exists a lower limit on the lightest neutrino mass if the size of perturbations is restricted. As it can be seen from eq. (4.5), this is a generic feature of obtained neutrino mass matrix independent of a choice of group parameters. From eq. (4.5), one obtains the 

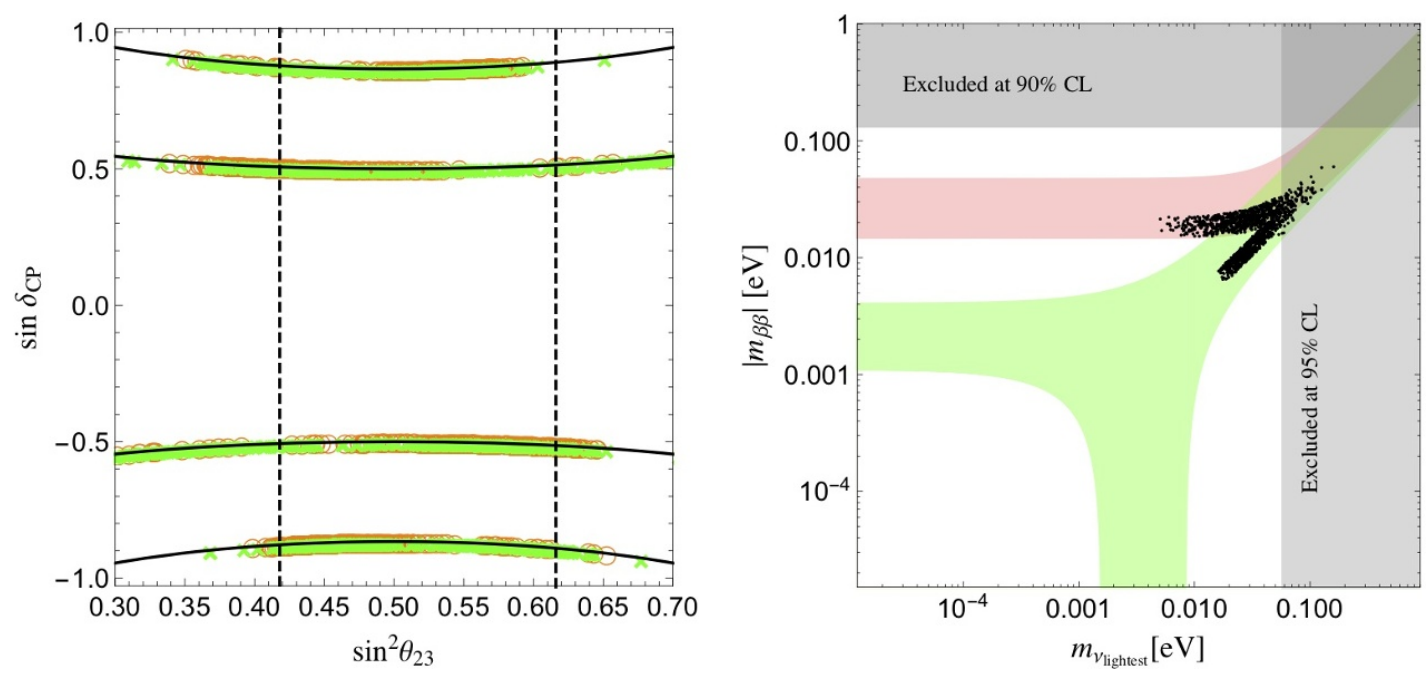

Figure 4. Same as figure 3 but for $n=6$ (with $-\eta_{1}=\eta_{2}=1, \gamma=1$ and $x=1,2$ ).

following expressions for the neutrino masses at the leading order in $\epsilon_{i}$ :

$$
\begin{aligned}
& m_{\nu_{1}}^{2}=m_{0}^{2}\left(A^{2}+B^{2}+\epsilon_{3} A^{2}\left(1-\sqrt{1+\frac{B^{2}}{A^{2}}}\right)+\mathcal{O}\left(\epsilon_{i}^{2}\right)\right), \\
& m_{\nu_{2}}^{2}=m_{0}^{2}\left(A^{2}+B^{2}+\epsilon_{3} A^{2}\left(1+\sqrt{1+\frac{B^{2}}{A^{2}}}\right)+\mathcal{O}\left(\epsilon_{i}^{2}\right)\right), \\
& m_{\nu_{3}}^{2}=m_{0}^{2}\left(C^{2}+\mathcal{O}\left(\epsilon_{i}^{2}\right)\right) .
\end{aligned}
$$

Note that splitting between $m_{\nu_{1}}$ and $m_{\nu_{2}}$ is induced by only $\epsilon_{3}$ at the leading order while $\epsilon_{1,2}$ contribute at the second order. The above masses imply the following relation between the solar squared mass difference and $m_{\nu_{1}}$.

$$
\Delta m_{21}^{2}=m_{\nu_{1}}^{2}\left(\frac{2 \epsilon_{3}}{\sqrt{1+B^{2} / A^{2}}}+\mathcal{O}\left(\epsilon_{i}^{2}\right)\right) .
$$

The above relation implies that $m_{\nu_{1}}$ cannot be arbitrary small for finite value of $\epsilon_{3}$ for nonzero $\Delta m_{21}^{2}$. For $\mathcal{O}(1)$ values of parameters $A$ and $B$, one obtains $m_{\nu_{1}} \gtrsim 0.05(0.015)$ $\mathrm{eV}$ for $\epsilon_{3} \lesssim 0.02(0.1)$ from eq. (5.2) which sets lower bound on the mass of the lightest neutrino in case of normal ordering. In case of inverted ordering, a lower bound on the lightest neutrino mass arises if $|C|>\left|\epsilon_{i}\right|$ is assumed as seen from eq. (5.1). The above observations are in agreement with the numerical results discussed earlier in this section.

\section{Summary}

Flavour symmetries with or without CP have been widely used for prediction of the leptonic mixing angles and phases. We have shown here that the flavour dependent CP symmetries can also play a role in restricting neutrino masses and can lead to a partially or fully degenerate neutrino spectrum. We have studied this in a general set up independent of 
any specific groups and discussed underlying constraints. The degeneracy follows from the conventional assumptions, eqs. (2.1), (2.3) made in the standard approaches [16, 17, 20] proposed to constrain neutrino mixing parameters. The only specific additional requirement is either eq. (2.5) or eq. (2.6) which imply degeneracy in the masses of two or three neutrinos respectively. We have elaborately discussed a case which leads to degenerate solar neutrino pair. The $\Delta\left(6 n^{2}\right)$ groups with even $n$ provide concrete examples of general set up discussed here. GenCP symmetries implying partially degenerate neutrinos within these groups were already discussed in [20] but the occurrence of two degenerate neutrinos within them was not noticed.

The symmetries envisaged here can also be used for predictions of the neutrino mixing angles and phases. However, a complete determination of these requires perturbations to break degeneracy in masses. We have discussed specific perturbations in $\Delta\left(6 n^{2}\right)$ which break the residual Klein symmetry of $M_{\nu}$ but preserve the underlying CP symmetry. Even after perturbations, the GenCP invariance of the full mass matrix $M_{\nu}$ leads to predictions of $\mathrm{CP}$ violating phases $\delta_{C P}, \alpha_{21}, \alpha_{31}$ in terms of a group theoretical phase factor and the atmospheric mixing angle $\theta_{23}$. The phenomenologically interesting scenario of residual generalized $\mu-\tau$ symmetry, which predicts maximal $\sin 2 \theta_{23}$ and $\delta_{C P}$, is obtained as a special case here. Detailed predictions of $\Delta\left(6 n^{2}\right)$ groups for $n=2,4,6$ are numerically studied taking into account constraints on neutrino masses and mixing parameters from the latest global fits to neutrino oscillation data. The smallest group $S_{4}$ predicts $\sin ^{2} \theta_{23}=1 / 2$ and $\delta_{C P}= \pm \pi / 2$, even in the presence of $\mathrm{CP}$ invariant perturbations. The same predictions can also be obtained by any of the groups in $\Delta\left(6 n^{2}\right)$ series with even $n$. The groups with higher order can lead to progressively smaller values of $\sin \delta_{C P}$ as well. For all the cases studied here, it is found that the smallness of the size of perturbations puts lower bound on the mass of the lightest neutrino. Therefore, the predictions made here can be tested not only from the precise measurements of atmospheric angle and Dirac CP phase but also from the experiments sensitive to the absolute scale of the neutrino masses.

\section{Acknowledgments}

This work was supported by BRNS (Department of Atomic Energy) and by Department of Science and Technology, Government of India through the Raja Ramanna fellowship and the J. C. Bose grant respectively. The work of KMP was partially supported by SERB Early Career Research Award (ECR/2017/000353) and by a research grant under INSPIRE Faculty Award (DST/INSPIRE /04/2015/000508) from the Department of Science and Technology, Government of India.

Open Access. This article is distributed under the terms of the Creative Commons Attribution License (CC-BY 4.0), which permits any use, distribution and reproduction in any medium, provided the original author(s) and source are credited.

\section{References}

[1] G. Altarelli and F. Feruglio, Discrete flavor symmetries and models of neutrino mixing, Rev. Mod. Phys. 82 (2010) 2701 [arXiv: 1002.0211] [InSPIRE]. 
[2] G. Altarelli, F. Feruglio and L. Merlo, Tri-bimaximal neutrino mixing and discrete flavour symmetries, Fortsch. Phys. 61 (2013) 507 [arXiv:1205.5133] [InSPIRE].

[3] A. Yu. Smirnov, Discrete symmetries and models of flavor mixing, J. Phys. Conf. Ser. 335 (2011) 012006 [arXiv:1103.3461] [INSPIRE].

[4] S.F. King and C. Luhn, Neutrino mass and mixing with discrete symmetry, Rept. Prog. Phys. 76 (2013) 056201 [arXiv: 1301.1340] [INSPIRE].

[5] H. Ishimori et al., Non-abelian discrete symmetries in particle physics, Prog. Theor. Phys. Suppl. 183 (2010) 1 [arXiv:1003.3552] [INSPIRE].

[6] D. Hernandez and A.Yu. Smirnov, Relating neutrino masses and mixings by discrete symmetries, Phys. Rev. D 88 (2013) 093007 [arXiv: 1304.7738] [INSPIRE].

[7] A.S. Joshipura and K.M. Patel, Discrete flavor symmetries for degenerate solar neutrino pair and their predictions, Phys. Rev. D 90 (2014) 036005 [arXiv: 1405.6106] [INSPIRE].

[8] L. Wolfenstein, Different varieties of massive Dirac neutrinos, Nucl. Phys. B 186 (1981) 147.

[9] S.T. Petcov, On pseudo-Dirac neutrinos, neutrino oscillations and neutrinoless double $\beta$-decay, Phys. Lett. B 110 (1982) 245.

[10] C.N. Leung and T. Petcov, A comment on the coexistence of Dirac and Majorana massive neutrinos, Phys. Lett. B 125 (1983) 461.

[11] C.S. Lam, Symmetry of lepton mixing, Phys. Lett. B 656 (2007) 193 [arXiv:0708.3665] [INSPIRE].

[12] C.S. Lam, Determining horizontal symmetry from neutrino mixing, Phys. Rev. Lett. 101 (2008) 121602 [arXiv:0804.2622] [INSPIRE].

[13] C.S. Lam, The unique horizontal symmetry of leptons, Phys. Rev. D 78 (2008) 073015 [arXiv:0809.1185] [INSPIRE].

[14] C.S. Lam, Finite symmetry of leptonic mass matrices, Phys. Rev. D 87 (2013) 013001 [arXiv: 1208.5527] [INSPIRE].

[15] C.S. Lam, Group theory and dynamics of neutrino mixing, Phys. Rev. D 83 (2011) 113002 [arXiv:1104.0055] [INSPIRE].

[16] F. Feruglio, C. Hagedorn and R. Ziegler, Lepton mixing parameters from discrete and $C P$ symmetries, JHEP 07 (2013) 027 [arXiv: 1211.5560] [INSPIRE].

[17] M. Holthausen, M. Lindner and M.A. Schmidt, CP and Discrete Flavour Symmetries, JHEP 04 (2013) 122 [arXiv: 1211.6953] [INSPIRE].

[18] C. Hagedorn, A. Meroni and E. Molinaro, Lepton mixing from $\Delta\left(3 n^{2}\right)$ and $\Delta\left(6 n^{2}\right)$ and $C P$, Nucl. Phys. B 891 (2015) 499 [arXiv:1408.7118] [InSPIRE].

[19] P. Chen, C.-C. Li and G.-J. Ding, Lepton flavor mixing and CP symmetry, Phys. Rev. D 91 (2015) 033003 [arXiv: 1412.8352] [INSPIRE].

[20] S.F. King and T. Neder, Lepton mixing predictions including Majorana phases from $\Delta\left(6 n^{2}\right)$ flavour symmetry and generalised CP, Phys. Lett. B 736 (2014) 308 [arXiv:1403.1758] [INSPIRE].

[21] G.-J. Ding, S.F. King and T. Neder, Generalised $C P$ and $\Delta\left(6 n^{2}\right)$ family symmetry in semi-direct models of leptons, JHEP 12 (2014) 007 [arXiv:1409.8005] [INSPIRE]. 
[22] I. Girardi, S.T. Petcov, A.J. Stuart and A.V. Titov, Leptonic Dirac CP-violation predictions from residual discrete symmetries, Nucl. Phys. B 902 (2016) 1 [arXiv:1509.02502] [INSPIRE].

[23] C.-C. Li, C.-Y. Yao and G.-J. Ding, Lepton mixing predictions from infinite group series $D_{9 n, 3 n}^{(1)}$ with generalized CP, JHEP 05 (2016) 007 [arXiv: 1601.06393] [INSPIRE].

[24] C.-Y. Yao and G.-J. Ding, CP symmetry and lepton mixing from a scan of finite discrete groups, Phys. Rev. D 94 (2016) 073006 [arXiv: 1606.05610] [INSPIRE].

[25] A.S. Joshipura, Perturbed flavour symmetries and predictions of CP-violating phase $\delta$, arXiv: 1801.02843 [INSPIRE].

[26] S.T. Petcov, Discrete flavour symmetries, neutrino mixing and leptonic CP-violation, arXiv:1711.10806 [INSPIRE].

[27] P. Chen, C.-Y. Yao and G.-J. Ding, Neutrino mixing from CP symmetry, Phys. Rev. D 92 (2015) 073002 [arXiv:1507.03419] [INSPIRE].

[28] C.-C. Li and G.-J. Ding, Implications of residual CP symmetry for leptogenesis in a model with two right-handed neutrinos, Phys. Rev. D 96 (2017) 075005 [arXiv:1701.08508] [INSPIRE].

[29] G. Ecker, W. Grimus and W. Konetschny, Quark mass matrices in left-right symmetric gauge theories, Nucl. Phys. B 191 (1981) 465.

[30] G. Ecker, W. Grimus and H. Neufeld, Spontaneous CP violation in left-right symmetric gauge theories, Nucl. Phys. B 247 (1984) 70.

[31] J. Bernabéu, G.C. Branco and M. Gronau, CP restrictions on quark mass matrices, Phys. Lett. B 169 (1986) 243.

[32] M.-C. Chen et al., CP violation from finite groups, Nucl. Phys. B 883 (2014) 267 [arXiv: 1402.0507] [INSPIRE].

[33] J.A. Escobar and C. Luhn, The flavor group $\Delta\left(6 n^{2}\right)$, J. Math. Phys. 50 (2009) 013524 [arXiv:0809.0639] [INSPIRE].

[34] S.F. King, T. Neder and A.J. Stuart, Lepton mixing predictions from $\Delta\left(6 n^{2}\right)$ family symmetry, Phys. Lett. B 726 (2013) 312 [arXiv:1305.3200] [INSPIRE].

[35] A.S. Joshipura and K.M. Patel, Residual $Z_{2}$ symmetries and leptonic mixing patterns from finite discrete subgroups of U(3), JHEP 01 (2017) 134 [arXiv:1610.07903] [INSPIRE].

[36] Particle Data Group, C. Patrignani et al., Review of particle physics, Chin. Phys. C 40 (2016) 100001.

[37] P. Chen, G.-J. Ding, F. Gonzalez-Canales and J.W.F. Valle, Generalized $\mu-\tau$ reflection symmetry and leptonic CP-violation, Phys. Lett. B 753 (2016) 644 [arXiv:1512.01551] [INSPIRE].

[38] W. Grimus and L. Lavoura, A Nonstandard CP transformation leading to maximal atmospheric neutrino mixing, Phys. Lett. B 579 (2004) 113 [hep-ph/0305309] [INSPIRE].

[39] T. Kitabayashi and M. Yasue, $\mu-\tau$ symmetry and maximal CP-violation, Phys. Lett. B 621 (2005) 133 [hep-ph/0504212] [INSPIRE].

[40] Y. Farzan and A. Yu. Smirnov, Leptonic CP-violation: zero, maximal or between the two extremes, JHEP 01 (2007) 059 [hep-ph/0610337] [INSPIRE]. 
[41] A.S. Joshipura, B.P. Kodrani and K.M. Patel, Fermion masses and mixings in a $\mu-\tau$ symmetric SO(10), Phys. Rev. D 79 (2009) 115017 [arXiv:0903.2161] [InSPIRE].

[42] S. Gupta, A.S. Joshipura and K.M. Patel, Minimal extension of tri-bimaximal mixing and generalized $Z_{2} \times Z_{2}$ symmetries, Phys. Rev. D 85 (2012) 031903 [arXiv:1112.6113] [INSPIRE].

[43] A.S. Joshipura and K.M. Patel, Generalized $\mu-\tau$ symmetry and discrete subgroups of $O(3)$, Phys. Lett. B 749 (2015) 159 [arXiv:1507.01235] [InSPIRE].

[44] H.-J. He, W. Rodejohann and X.-J. Xu, Origin of constrained maximal CP-violation in flavor symmetry, Phys. Lett. B 751 (2015) 586 [arXiv: 1507.03541] [INSPIRE].

[45] Z.-z. Xing and Z.-h. Zhao, A review of $\mu$ - $\tau$ flavor symmetry in neutrino physics, Rept. Prog. Phys. 79 (2016) 076201 [arXiv: 1512.04207] [INSPIRE].

[46] I. Esteban et al., Updated fit to three neutrino mixing: exploring the accelerator-reactor complementarity, JHEP 01 (2017) 087 [arXiv:1611.01514] [INSPIRE].

[47] P. Guzowski et al., Combined limit on the neutrino mass from neutrinoless double- $\beta$ decay and constraints on sterile Majorana neutrinos, Phys. Rev. D 92 (2015) 012002 [arXiv: 1504.03600] [INSPIRE].

[48] F. Couchot et al., Cosmological constraints on the neutrino mass including systematic uncertainties, Astron. Astrophys. 606 (2017) A104 [arXiv:1703.10829] [INSPIRE]. 